\title{
A Wearable, Low-cost Hand Tremor Sensor for Detecting Hypoglycemic Events in Diabetic Patients
}

\begin{abstract}
Severe hypoglycemia leverages complication in diabetes patients: e.g., it increases death rate by a six-fold. Therefore, early detection and prediction of hypoglycemic events are of utmost importance. This publication presents a prototype of a wearable hand-tremor system that detects the onset of hypoglycemic events. The results show the prototype is capable of simulating anticipated frequency and amplitude of the tremor relevant for hypoglycemic events. The initial functional performance-tests demonstrate a maximum error of $4.75 \%$ in the detecting the tremor frequency.

Index Terms-Hypoglycemia, Diabetes, Hand tremor, Wearable sensor, Internet of Things
\end{abstract}

\section{INTRODUCTION}

Hypoglycemia is a condition with the blood glucose level below the threshold of $70 \mathrm{mg} / \mathrm{dL}$ [1] and is considered serious adverse effect of insulin based treatment for type1 diabetes (T1D) patients. In a hypoglycemic state, the human body of a healthy individual initiates a variety of reactive measures such as suppression of insulin secretion and limiting glucose utilization, which is analogous to low-battery mode in modern day electronic communication devices. This results in an auto-regulatory response to stabilize the system: e.g. seeking for food. However, for a T1D patient, this response is unstable and potentially develops into a state of hypoglycemia [2]. A recurrent and prolonged hypoglycemia possibly even leads to impairment of the counter-regulatory system with the potential for development of hypoglycemia unawareness. In addition to health-related quality-of-life issues (sleep, driving, employment), the short-term complications include neurocognitive dysfunction, temporal loss of vision, and may result in eventual loss of life [3]. The longterm complications include retinal cell death, cardiovascular complications, i.e., myocardial infarction. Therefore, early detection and prediction of hypoglycemic events is of utmost importance.

One of the earliest manifestations of hypoglycemia is the involuntary shaking of the human body such as the fingertips [4]. Although the tremor can be attributed to a variety of physiological phenomena (e.g. neurological disease), the tremor associated specifically with hypoglycemia is categorized as Enhanced Physiological Tremor and situated in the frequency range of 10-14 Hz [5]. However, the majority of studies focus on the other physiological factors such as sweat and body temperature. With environmental factors greatly influencing the skin conductance and body temperature, these parameters are not accurate predictors of hypoglycemia [6]. Furthermore, our literature search revealed very few studies using hypoglycemic tremors or utilized technology to correlate tremors with the onset of hypoglycemia [7]. One study attempted to detect hypoglycemia through tremors as a continuous movement for more than 30 seconds in the wrist without specific analysis of the tremor characteristics. However, tremors in an index finger showed a significant change with dropping blood glucose levels [8]. Concurrently, finger tremor was found to be more resilient against becoming impaired like adrenaline responses [8].

In this study, we present an accelerometer-based prototype of a tremor sensor assessing the frequency of hypoglycemia. This wearable system aims to achieve clinical accuracy necessary during hypoglycemia camp-experiments.

\section{STUdy OVERVIEW}

The proposed concept, shown in Fig. 1, is an internet of things (IoT) consisting of:

- a sensor mounted on the index finger: chargeable and equipped to wirelessly transmit the accelerometer data,

- a smart wrist band: collects and processes the data in real-time, and notifies to caregiver by push notifications,

- a cloud computing platform equipped with machine learning algorithm for data aggregation.

Additionally, a smart wristband is also a front-end hat alerts the user in the event of a hypoglycemia episode. Haptic feedback issued to the wearable system on the wrist offers a secondary mode of alerting the user.

\section{EXPERIMENTAL PROTOCOL}

\section{A. Experiment 1}

To validate the concept, we designed an experiment illustrated in Fig. 2. A triple axis accelerometer (ADXL 335) was mounted on a speaker-based mechanical wave generator (Pasco SF-9324) and powered by a sine wave created by a function generator. The accelerometer was connected to a microcontroller powered circuit (Adafruit Feather $n R F 52$ Bluefruit $L E$ ) and the sensor data was logged on a personal computer via a serial link. In order to simulate hand tremors attributed to hypoglycemia, the frequency of the sine wave was varied from $8 \mathrm{~Hz}$ to $14 \mathrm{~Hz}$ in $2 \mathrm{~Hz}$ steps. The amplitude of the mechanical wave was also varied by changing the voltage of the electrical signal from $10 \mathrm{mV}$ to $2 \mathrm{~V}$. In addition, we recorded the hand tremors which were induced when a subject fatigued his dominant hand through holding a dynamometer, at persistent maximum grip strength. 


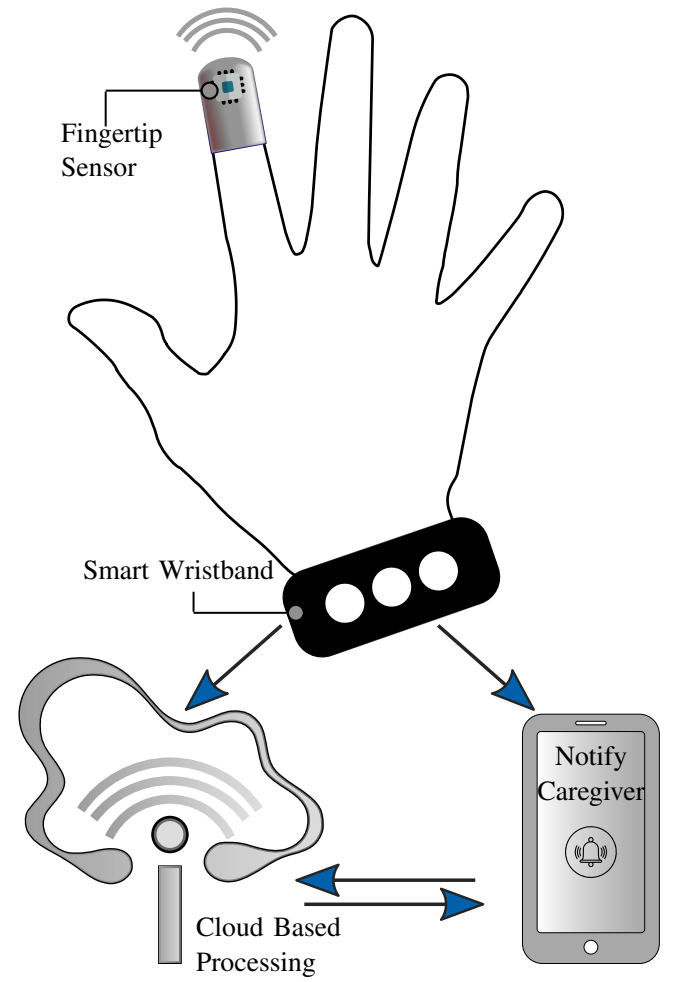

Fig. 1: Project overview illustrating fingertip sensor with accelerometer. Data is collected and processed in real-time in a smart wristband. Caregivers are notified by push messages on smartphones. Hypoglycemic events are detected using machine learning algorithms and the user is alerted on the phone and wristband microcontroller.

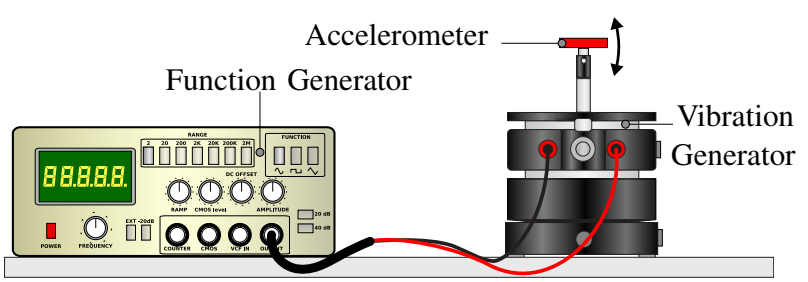

Fig. 2: Illustration of the accelerometer sensitivity measurement setup.

\section{B. Experiment 2}

In the second experiment, the amplitude of the mechanical wave was measured, in terms of the arm displacement, as a function of the electrical signal voltage. The displacement was generated by varying the frequency of the electrical signal from $8 \mathrm{~Hz}$ to $14 \mathrm{~Hz}$ in steps of $2 \mathrm{~Hz}$. For each frequency, the voltage was varied from $10 \mathrm{mV}$ to $2 \mathrm{~V}$ in ten steps. The mechanical wave amplitude was measured by an internal micrometer.

\section{RESUlts AND Discussion}

In Fig. 3, the recorded waveforms for two sine waves having frequency/voltage of $14 \mathrm{~Hz} / 1 \mathrm{~V}$ and $10 \mathrm{~Hz} / 150 \mathrm{mV}$ respectively are shown. The low voltage curve is enhanced by a factor of 10. Moreover, Fig. 3 also shows the waveforms (yellow and purple) of the hand tremor induced at maximum hand grip strength. The corresponding frequency plots are shown in Fig. 4 for the four signals. Notably, the frequency content of the hand tremors lies in the range of 8 to $15 \mathrm{~Hz}$, while peaking at around $12 \mathrm{~Hz}$ for both recordings.

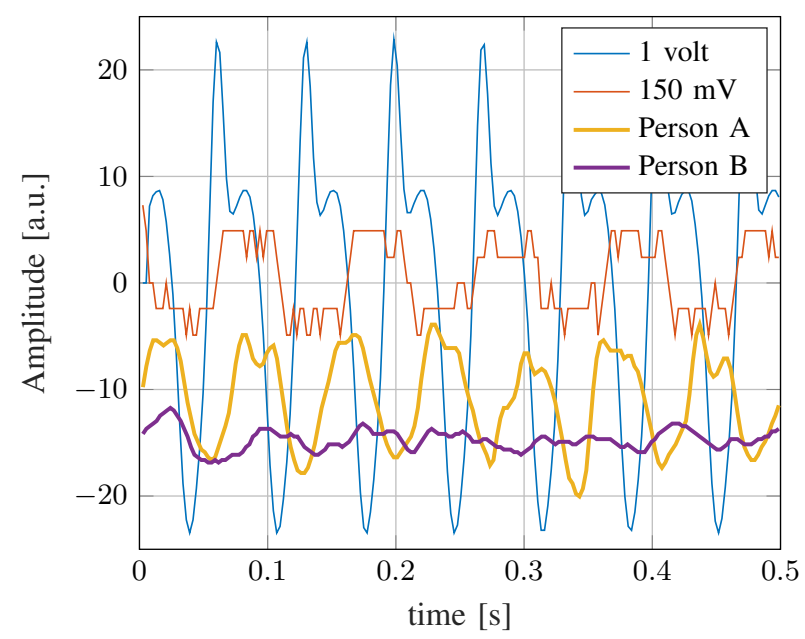

Fig. 3: Raw accelerometer data recorded on a personal computer. Blue curve is $14 \mathrm{~Hz}$ with a $1 \mathrm{~V}$ amplitude while the orange curve is $10 \mathrm{~Hz}$ and $150 \mathrm{mV}$. Yellow and purple curves are tremor waveforms induced after fatiguing the subject.

The difference between generated and measured frequencies (system error) originates from non-uniform transmission rate at the microcontroller. The error is shown in Fig. 5. In

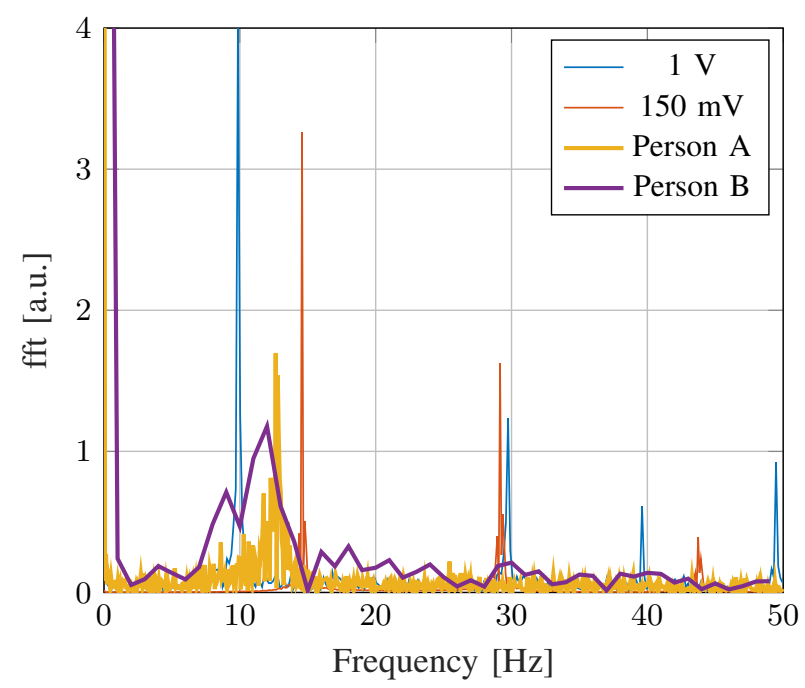

Fig. 4: Frequency content of the signals showing the dominant frequencies and their respective harmonics. For hand tremors, the frequencies lie in $8-15 \mathrm{~Hz}$ range.

the second experiment, a linear relationship was observed between the amplitude of the mechanical wave and the voltage of its powering electrical signal (Fig. 6). The results show that this mechanical drive is capable of simulating weak vibrations with a low amplitude $(0.1 \mathrm{~mm})$ similar to what has been reported in [9] on the relationship between essential tremor frequency and its amplitude.

\section{Conclusion and Future Perspectives}

This paper presents a low-cost and wearable prototype of a hand tremor sensing device capable of detecting low am- 


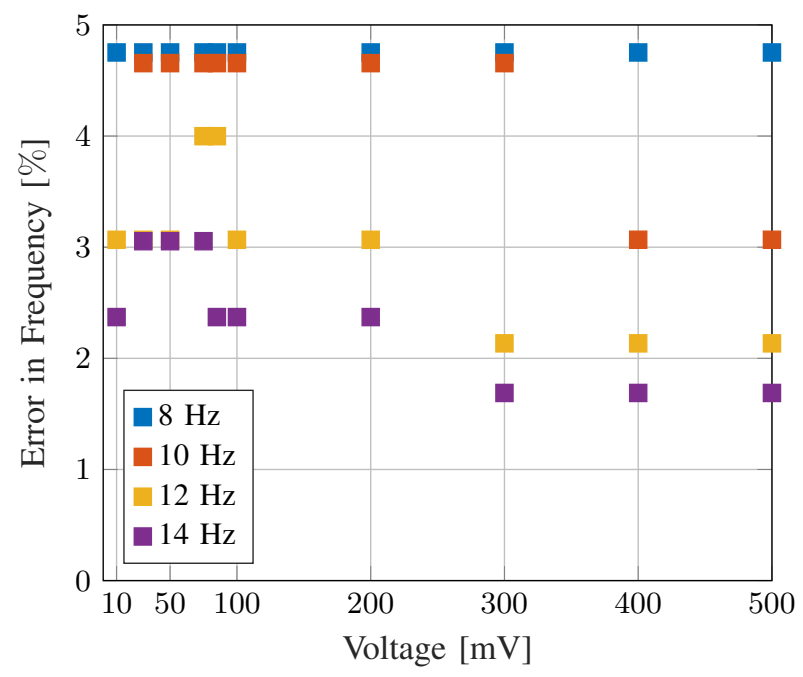

Fig. 5: Percentage error in the measured and configured frequencies.

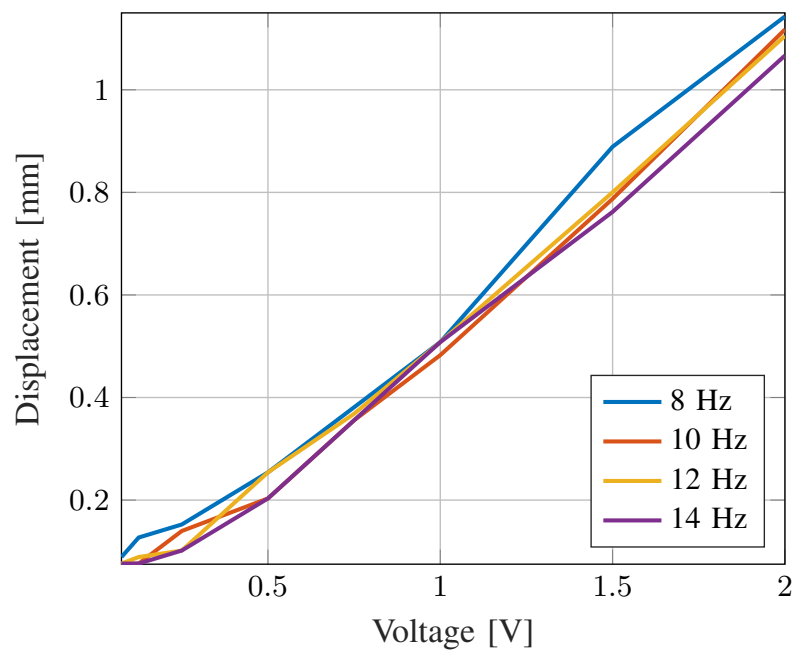

Fig. 6: Displacement of the vibration generator arm, specifying the mechanical wave amplitude as a function of voltage.

plitude tremor in a frequency range of $10-14 \mathrm{~Hz}$ attributed to hypoglycemia. The performance of the accelerometer-based will be validated on a group of diabetic patients and healthy volunteers in various age groups [10]. The validated device will be placed on human subjects during a hypoglycemic clamp experiment [11] under careful supervision of an experienced physician. During this procedure, the blood glucose level of a participant will be maintained at a predetermined values (less than $60 \mathrm{mg} / \mathrm{dL}$ ) by infusing insulin and glucose intravenously. The blood glucose level will be measured at 5 min intervals throughout the 60 min experiment.

\section{ACKNOWLEDGMENT}

This publication was made possible by NPRP grant number NPRP 10-1231-160071 from the Qatar National Research Fund (a member of Qatar Foundation). The statements made herein are solely the responsibility of the authors.

\section{REFERENCES}

[1] American Diabetes Association Workgroup on Hypoglycemia, "Defining and Reporting Hypoglycemia in Diabetes: A report from the American Diabetes Association Workgroup on Hypoglycemia," Diabetes Care, vol. 28, no. 5, pp. 1245-1249, May 2005.

[2] R. McCrimmon, "Glucose Sensing During Hypoglycemia: Lessons From the Lab," Diabetes Care, vol. 32, no. 8, pp. 1357-1363, Aug. 2009.

[3] R. G. McCoy, H. K. Van Houten, J. Y. Ziegenfuss, N. D. Shah, R. A. Wermers, and S. A. Smith, "Increased Mortality of Patients With Diabetes Reporting Severe Hypoglycemia," Diabetes Care, vol. 35, no. 9, pp. 1897-1901, Sep. 2012.

[4] I. Muhlhauser, L. Heinemann, E. Fritsche, K. von Lennep, and M. Berger, "Hypoglycemic Symptoms and Frequency of Severe Hypoglycemia in Patients Treated With Human and Animal Insulin Preparations," Diabetes Care, vol. 14, no. 8, pp. 745-749, Aug. 1991.

[5] A. Q. Rana and K. L. Chou, Essential Tremor in Clinical Practice, ser. In Clinical Practice. Cham: Springer International Publishing, 2015.

[6] D. Howsmon and B. W. Bequette, "Hypo- and Hyperglycemic Alarms: Devices and Algorithms," Journal of Diabetes Science and Technology, vol. 9, no. 5, pp. 1126-1137, Sep. 2015.

[7] K. Zahed, F. Sasangohar, Y. Zhou, R. Mehta, M. Erraguntla, and M. Lawley, "Investigating the Efficacy of Using Hand Tremors for Early Detection of Hypoglycemic Events: A Scoping Literature Review," in Proceedings of Human Factors and Ergonomics Society, Philadelphia, PA, 2018.

[8] E. George, N. Harris, C. Bedford, I. A. Macdonald, C. A. Hardisty, and S. R. Heller, "Prolonged but partial impairment of the hypoglycaemic physiological response following short-term hypoglycaemia in normal subjects," Diabetologia, vol. 38, no. 10, pp. 1183-1190, Oct. 1995.

[9] S. Calzetti, M. Baratti, M. Gresty, and L. Findley, "Frequency/amplitude characteristics of postural tremor of the hands in a population of patients with bilateral essential tremor: implications for the classification and mechanism of essential tremor." Journal of Neurology, Neurosurgery \& Psychiatry, vol. 50, no. 5, pp. 561-567, 1987.

[10] Y. Zhou, K. Zahed, R. Mehta, F. Sasangohar, M. Erraguntla, and M. Lawley, "Non-invasive Wearable System for Hypoglycemia Detection: A Proof of Concept User-Centered Design Process," in Proceedings of Human Factors and Ergonomics Society, Philadelphia, PA, 2018.

[11] S. L. Cichosz, J. Frystyk, O. K. Hejlesen, L. Tarnow, and J. Fleischer, "A novel algorithm for prediction and detection of hypoglycemia based on continuous glucose monitoring and heart rate variability in patients with type 1 diabetes," Journal of Diabetes Science and Technology, vol. 8, no. 4, pp. 731-737, Jul. 2014. 